\title{
National Conference on Parkinson's Disease
}

\author{
A.H. Rajput (University of Saskatchewan) \\ D. Calne (University of British Columbia) \\ A.E. Lang (University of Toronto)
}

\begin{abstract}
A National Conference on Parkinson's disease was held on September 7th-8th, 1990 in Victoria, British Columbia.The scientific program included 11 formal presentations, 10 small group workshops and video presentations of interesting examples of movement disorders. The subjects discussed ranged from epidemiology and etiology to current and possible future modes of management of Parkinson's disease.
\end{abstract}

Epidemiology and etiology of Parkinson's disease was discussed by Dr. A. Rajput of University of Saskatchewan.

Parkinson syndrome (PS) is characterized by bradykinesia, rigidity and resting tremor -2 of the 3 are necessary for the diagnosis. There is no readily available laboratory test which is diagnostic of PS. The PS is not a single disease entity but a group of disorders that have similar clinical features. In the majority of PS there is no known cause and hence they are "idiopathic" disorders. However, most neurologists use the terms idiopathic Parkinson's disease and Parkinson's disease (PD) for only those cases that are presumed to have substantia nigra (SN) neuronal loss and Lewy body (LB) inclusions. Since the definite diagnosis of PD depends on pathological observations, clinical distinction between PD and other variants of PS is not always possible. In the early cases where the diagnosis of PD was made by a neurologist and patients were followed for several years, between $1 / 4$ to $1 / 3$ did not have LB pathology. The response to drug therapy in the other variants of PS may be similar to that in the PD cases. Additionally, some cases of drug induced parkinsonism may have underlying LB pathology. Because of our inability to clinically distinguish different pathological variants of PS, especially during early stage, and the fact that all forms of PS produce disability which requires treatment, for the purpose of descriptive epidemiological studies, all Parkinson syndrome cases in the general population should be included.

The annual incidence of PS in the North American general population is estimated at 20.5 per 100,000 and the mean survival in the Saskatoon series is slightly more than 13 years. The minimum estimated prevalence rate of PS is 270 per 100,000 in the North American population. Approximately 5\% cases have the onset before age 40 years. The incidence and the prevalence of PS increase with advancing age. The prevalence rate is $1 \%$ in the population over 60 years and it increases to $2.5 \%$ in those over age 85 years. Because the proportion of older population is slowly increasing, we should expect a higher prevalence rate of PS in the future. A very large proportion of the PS in the elderly goes unrecognized. In Mississippi and in Saskatoon $40 \%$ of the elderly PS were undiagnosed. The survival in the PS since the introduction of levodopa in 1967 has improved. When the survival was measured from the date of the onset of PS and compared retrospectively with the survival life tables in the general population, no significant difference was noted. That, however. would need further verification using more stringent methods comparing the life expectancy in the PS cases with the general population.

The pursuit of etiology has been concentrated on PD which accounts for more than $80 \%$ of PS. There are two main theories about the cause of PD - genetic and environmental.

In three different studies where one twin had PD, the risk of $P D$ in the other twin was not significantly higher than the general population. The prevalence of PD among blacks in the United States is five times higher than in Nigerian blacks, but the prevalence rate in blacks and whites living in a Mississippi community is similar. Isolated pedigrees of clinically atypical familial LB disease have been reported but, on balance, the evidence strongly favours environmental cause(s) for PD. Genetic predisposition in rare cases may play a role in the etiology.

The search for a specific environmental cause is difficult because the disease usually manifests around age 60 and many prior events could play a role. By restricting the search to only those PD cases who had onset of motor manifestations before age 40 Rajput and colleagues noted that nearly every case in their series was born and raised in rural Saskatchewan. They later performed SN neuronal counts in neurologically normal cases and noted that residents in rural areas had fewer SN neurons compared to urban residents. Well water used in rural communities should be considered as a vehicle for the etiological agent in future studies of PD. 
Dr. J. Kalra from the University of Saskatchewan spoke on Pathogenesis: Oxygen free radicals in Parkinson's disease. He summarized some of the potential endogenous causative factors including oxygen free radicals.

Oxygen free radicals (superoxide anion, hydrogen peroxide and hydroxyl radical) are produced by the univalent reduction of oxygen. Normally the free radicals are removed by different scavenger/catalytic systems present in the blood and tissue. Oxygen fee radicals (OFR) have been implicated as contributing factors to pathogenesis in a variety of biological systems as well as in PD. An increase in the levels of OFR productions, however, has not been conclusively demonstrated in this condition so far.

There are various sources of OFR including activation of polymorphonuclear (PMN) leukocytes which initiate a "respiratory burst" with a sudden and large increase in OFR that can be released into the external environment. Superoxide anion $\left(\mathrm{O}_{2}{ }^{-}\right)$ thus formed by the univalent reduction of oxygen reacts to form hydrogen peroxide $\left(\mathrm{H}_{2} \mathrm{O}_{2}\right)$, a reaction that is catalyzed by superoxide dismutase (SOD). The formation of hydroxyl radical (.OH) requires the reaction between $\mathrm{O}_{2}-$ and $\mathrm{H}_{2} \mathrm{O}_{2}$.

The OFR exert cytotoxic effects by causing peroxidation of membrane phospholipids which would result in increased fluidity leading to enhanced permeability and loss of cell membrane integrity.

If the OFR play a role in the pathogenic process of $\mathrm{PD}$, then there may be an increase in oxygen free radical producing activity of PMN leukocytes and in the contents of the lipid peroxidation product the serum malondialdehyde (MDA).

Dr. Kalra and his colleagues investigated the oxygen free radical producing action of PMN leukeocytes in the blood and MDA in the serum from the PD patients and normal subjects. The controls included 39 healthy hospital workers (mean age $30.1 \pm 1.5$ ) who were not taking any medication and $22 \mathrm{PD}$ patients (mean age $59.4 \pm 2.1$ ) None of the PD cases were on symptomatic antiparkinsonian drugs. Venous blood samples from each subject were collected in vacutainer tubes containing EDTA. The PMN leukocyte counts were obtained using a Technicon 6000 and by microscopic differential. The OFR activity of PMN leukocytes was measured using luminol dependent chemiluminescence. Serum MDA was determined using thiobarbituric acid (TBA) reagent.

The total OFR producing activity (chemiluminescence) of PMN leukocytes in the blood from PD patients was significantly higher than in the normal subjects. Also there was a marked increase in the SOD-inhibitable chemiluminescent activity of PMN leukocytes in PD as compared to control subjects. Serum MDA levels in PD patients were significantly higher than in the normal subjects. These data indicate that PMN leukocytes of patients with PD have increased capacity to produce oxygen free radicals and that the lipid peroxidation is increased in patients with PD.

Dr. Donald Calne from the University of British Columbia reviewed progress in the field of dopamine receptors during the last five years. He laid particular emphasis on techniques of molecular biology in analyzing the structure of dopamine receptors (DR). He discussed the six looped polypeptide structure with seven transmembrane domains which has been shown to be the receptor molecule and gave details of the structure for both
D1 and D2 receptors. The two variants of this structure have been found for both the D1 and D2 receptors. The difference between them depend upon an insert of amino acids in each case. The D2 receptor is made up of a chain of 415 and 444 aminoacids and its two isoforms. The DI receptor is of similar size and configuration.

The dopamine receptors (DR) are classified into the category of "Class II" receptors as opposed to Class I receptors. The latter are ligand binding subunits linked directly to an ion channel and they react very rapidly to activation. Class II receptors are slower to respond than Class I receptors and are subject to a more complex control mechanisms that includes potential for amplification, attenuation, and continuous modulation according to circumstances. These features of Class II receptors are achieved through linkage of the receptor to guanine nucleotide regulatory proteins (G-proteins) acting as transducers to an effector system such as adenylyl cyclase. The receptor-G-proteineffector array is employed for many different categories of neurotransmitter receptors.

While advances in the field of receptor pharmacology over the last few years have been quite dramatic from the academic viewpoint, they are also beginning to establish a basis of potential therapeutic importance. The control of receptor mediated events by the complex mechanisms that are now being identified should allow selective manipulation with drugs. Thus the practical impact of these theoretical advances could enable antiparkinsonian agents to be developed with fewer side effects and greater efficacy.

Dr. D. Grimes from the University of Ottawa, discussed some important non-pharmalogical measures in the management of PD. Over the past six years it has become accepted that protein intake decreases the effects of levodopa and protein restriction benefits more than half of patients with motor fluctuations. Levodopa transport into the brain is inhibited by large neutral amino acids (LNAA) in the diet. Protein restriction reduces the LNAA competition for levodopa transport. It is possible that such dietary manipulations may also induce receptor or metabolic changes and thus improve levodopa effectiveness. An average North American diet contains $1.6 \mathrm{gm} / \mathrm{Kgm} /$ day of protein but only half that amount is essential.

A trial of protein restriction is indicated in a patient who has motor fluctuations which do not respond to the usual pharmacological manipulations. Levodopa non-responders on the other had, do not show a consistent response to protein restrictions. Those that improve will show reduction in the "off" time, but the quality of "on" time remains unchanged. General guidelines for a regime of protein restriction include: 1) A dietician must be involved. 2) The diet must be tasty to reduce non-compliance. 3) Reduced protein over the three meals or protein only at supper are the therapeutic choices. 4) The patient must not lose weight.

Approximately $60 \%$ of patients will achieve some benefit on protein restriction and the response is usually evident within one week. If the "protein at evening meal diet", is selected, patients usually do not become worse by the evening. The most common side effects are increased peak dose dyskinesias or confusion which may require reduction in levodopa dosage. In one series, 3 of 16 cases who improved, otherwise became depressed requiring discontinuation of the diet. Benefit has been reported up to 16 months in some cases. A number of Dr. Grime's clinic 
patients have reported continued benefit from a modified protein restriction regime for three years. If the diet is adhered to, the benefit appears to persist.

The most common protein adjusted diet includes $7 \mathrm{gm}$ protein at breakfast and lunch and an unlimited protein at supper. Unlimited supper and evening protein would ensure a minimum daily intake of $0.8 \mathrm{mg} / \mathrm{kg}$ day.

Depression is a common feature in PD. There is now considerable evidence that treatment with electroconvulsive therapy (ECT) may improve both the depression and the parkinsonism. Improvement in motor deficits may occur even if depression is not a prominant feature, suggesting a direct antiparkinsonian effect of ECT. ECT treated PD patients may develop increased dyskinesias but reduced motor fluctuations.

The duration of motor improvement has been variable and sometimes disappointing. Improvement has varied from two weeks to eight months. The older patients with long duration levodopa therapy have the best and most sustained motor improvement. Patients who respond have improvement in all manifestations of PD - tremor, rigidity, bradykinesia, gait and postural instability as well as motor fluctuations.

Mania is another indication for the use of ECT. Dopaminomimetic psychosis is a treatment limiting problem in chronic levodopa and dopamine agonist therapy. Reduction in the medication often results in immobility and standard neuroleptics worsen parkinsonism. Clozapine and remoxipride, D2 blocking agents with limited striatal effect, have undergone clinical trials but the role of these drugs is not yet clear. ECT therapy has given excellent, sustained relief of psychosis in some patients, thus allowing maintenance of dopaminomimetic therapy. The adverse effects of ECT in Parkinson's disease patients have been minimal. Transient confusion is observed frequently and dyskinesias may increase after ECT. The ECT induced cognitive defect usually clears within a few days but in the demented patients it may become persistent. Dementia is therefore a contraindication to the use of ECT in PD. The use of unilateral, brief-pulse, right hemisphere ECT gives fewer cognitive side effects and is the preferred treatment option. Modern anesthetics, muscle relaxants and electrocardiographic monitoring have significantly reduced the risk and incidence of skeletal and cardiac adverse effects.

In summary, ECT may be considered for the following groups of Parkinson's disease patients.

1) Depression, not responsive to standard pharmacological therapy. 2) Psychotic disturbances and mania, which limit or complicate dopaminomimetic therapy. 3) Unresponsive motor fluctuations in selected older patients with long duration levodopa treatment. (This type of patient requires more study).

Dr. M. Muenter of the Mayo Clinic discussed Early symptomatic therapy: advantage and limitations.

When selecting drugs for the early symptomatic therapy of Parkinson's disease, one should take into account not only their relative long-term efficacy and side effects but also their long term effects upon longevity and disease progression.

Anticholinergics: Undoubtedly effective medications, their capacity to improve symptoms is maximally in the $30 \%$ range. They commonly cause impairment of memory, and less often confusion, hallucinations and urinary retention.
Amantadine: Therapeutically quite erratic, some patients experience side effects with no real beneficial effects, while others tolerate the medication and experience moderate improvement which either endures or fades away after a few months.

Dopamine agonists: In general, bromocriptine and pergolide are more effective than anticholinergics or amantadine. However, while monotherapy with agonists early in the disease is not as effective as carbidopa/levodopa, they do have an important role in the treatment of patients who have developed a short duration response to carbidopa/levodopa with on-off phenomena, particularly if accompanied by biphasic dyskinesias. Moreover, combined early treatment with carbodopa/levodopa and an agonist has been claimed to prevent the eventual occurrence of the onoff effects with carbidopa/levodopa later during the disease, but this hypothesis remains unproven.

Deprenyl: In monotherapy, it has been found to be ineffective or only slightly effective in early Parkinson's disease, but Deprenyl may be combined with carbidopa/levodopa because of its potential to slow progression of early disease. It also reduces dosage of the latter drug by $18 \%$ on average.

Carbidopa/levodopa: Early treatment with carbidopa/levodopa usually results in a long duration therapeutic response without any fluctuations or on-off effects. In many patients this response is maintained for five to ten years before it is gradually replaced by a short duration response. Because of its efficacy and favourable side effect profile, and its ability to improve life expectancy when administered early in the course of the disease, it is the drug of choice for the early treatment of Parkinson's disease.

Dr. Tom Hutton of Texas University reviewed his experience with controlled release Sinemet (Sinemet CR) preparation. Levodopa in combination with a dopa decarboxylase inhibitor (carbidopa or benserazide) is the primary treatment for the motor manifestations of PD and is highly effective for several years in most patients. Unfortunately, approximately $50 \%$ of patients treated for more than five years develop fluctuations in motor response. Motor response fluctuations usually begin with end-of-dose failure ("wearing off") and may progress to dyskinesias and rapid switching between periods of good mobility and relative immobility or dyskinesias ("on-off") The management of motor response fluctuations presents the greatest challenge in the treatment of advance PD.

Increasing the frequency of smaller individual doses of levodopa is traditionally employed for controlling the fluctuations. The effectiveness of this method is limited. Maintaining stable plasma levodopa concentrations through continuous intravenous and intraduodenal infusion may reduce or eliminate fluctuating motor response. While neither of these are practical routes of levodopa administration, this line of research provides a strong rationale for the development of a controlled release preparation which provide more stable levodopa levels than the short-acting, oral preparations currently marketed - Sinemet and Prolopa.

Better control of motor response fluctuations has been the overriding goal in the lengthy process to develop a controlled release form of carbidopa/levodopa. Sinemet $C R$ is the fourth generation of controlled release carbidopa/levodopa to be tested in clinical trials. It contains $50 \mathrm{mgs}$. of carbidopa and $200 \mathrm{mg}$. of levodopa in an erodable matrix that releases the active drugs over 4-6 hours. In a recent study, Dr. Hutton and colleagues 
compared the safety and efficacy of Sinemet CR (50/200) and standard Sinemet $(25 / 100)$ in a double-blind, crossover design. Two hundred and two patients with advanced PD, 132 males and 70 females, median age 65 years, from eight centers in the United States entered the trial. The average duration of PD was 10 years and all patients had experienced motor response fluctuations for 3.5 years, prior to the trial. 156 patients completed the double-blind phase.

They found that daily dosing frequency was reduced by a median of $33 \%$ with Sinemet CR (mean -6.8 per day). There was a median $25 \%$ increase in the daily levodopa - Sinemet CR (mean $-1238 \mathrm{mg}$ ) over the standard Sinemet dose (mean $975 \mathrm{mg}$ ). During double blind treatment both on physician and patient global ratings, the Sinemet CR patients had significant improvement compared to those treated with standard Sinemet. Analysis of patient motor fluctuation diaries revealed an average decrease in "off" time while on treatment with Sinemet CR as compared to standard Sinemet treatment. The percent of the waking day "off" state assessed by the Unified Parkinson's Disease Rating Scale, indicated a significant difference in favor of Sinemet CR at each evaluation. There was no significant difference in the adverse experiences on the two preparations. Of the patients stating a preference, Sinemet CR was preferred by $55 \%$ as compared to $27 \%$ preferring the standard Sinemet. One hundred forty-seven of the 156 patients who completed the double-blind study elected to continue Sinemet CR therapy.

Twelve patients who elected open-label Sinemet CR treatment extension have been followed for an additional 36 months. Standardized measures of motor function have shown gradual decline as would be expected in advanced PD cases. Nevertheless, analysis of patient diaries of daily motor response has been encouraging in that the ratios of time "on", "on with dyskinesias" and "off" have shown very little change over time. Dr. Hutton reported that they have seen no evidence that the salutary effect of Sinemet CR has diminished during this long-term extension period. He concluded that Sinemet CR is better than standard Sinemet for patients with advanced Parkinson's disease. The reduced dosing frequency required with Sinemet CR allows for more convenient dosing schedules, and may improve patient compliance and dosing titration. "Off" time is reduced by approximately 30-40 minutes per day on Sinemet CR as compared to Sinemet $25 / 100$. The motor fluctuations do attenuate, but continue to be apparent on Sinemet CR. While Sinemet CR does not solve the problem of fluctuating motor performance, Dr. Hutton regards it as a welcome new treatment for advanced Parkinson's disease.

Dr. M. Mouraidian of the National Institute of Neurological Disorders and Stroke, USA discussed Pharmacokinetic considerations in the management of PD. The initial, often dramatic, stable motor response to levodopa frequently, after a few years, gives way to a fluctuating state, characterized by wearing off and the later in some, the unpredictable on/off phenomenon. Peripheral pharmacokinetic factors such as altered gastric motility, short plasma half-life, and competition with dietary large neutral amino acids across the blood-brain barrier, all contribute to the motor fluctuations in advanced PD. However, these cannot explain the entire clinical picture. This is supported by the fact that patients with complex on/off problems continue to fluctuate despite constant plasma levels of L-dopa and restriction of protein intake. Central pharmacodynamic factors may thus play a crucial role. In advanced PD where normal storage and release of dopamine by presynaptic dopaminergic neurons is lost, postsynaptic receptors are exposed to oscillating or pulsed stimulation due to the periodic oral intake of medication. Chronic exposure of postsynaptic receptors to this nonphysiologic intermittent stimulation may result in steepening of the dose-response curve for levodopa and narrowing of the therapeutic window between toxic dyskinesias and beneficial antiparkinson effects, clinically evident as on/off fluctuations. Importantly, these indices of altered responsiveness demonstrate partial plasticity indicated by a gradual amelioration of on/off motor fluctuations after several days of continuous, round-the-clock dopaminomimetic therapy. Thus the work from this group has emphasized the combined role of disease progression and our current use of "pulsed" levodopa therapy in the late-stage problems of Parkinson's disease. Continuous dopaminomimetic treatment may both improve response fluctuations and even have a prophylactic value in delaying their onset or minimizing their severity.

The concept of continuous dopamimergic stimulation was further pursued by Dr. Jacob Sage from UMDNG-Robert Wood John Medical School, USA. The rationale for this approach is the evidence that one can ameliorate severe motor fluctuations and prevent worsening of the fluctuations with certain forms of continuous dopaminergic stimulation. He reviewed the clinical stages of response fluctuations from a stable response through to the stage of very short but predictable and later to unpredictable fluctuations. Importantly, overt motor fluctuations may appear even in the early phase of treatment and some believe that they are present in the majority of cases when carefully looked for. In addition to fluctuating symptomatology, patients may have a wide variety of dyskinesias, particularly chorea and dystonia. The relationship of these dyskinesias to plasma and thus the brain dopamine levels is complex. Some occur at peak dose, others during trough levels and still others at the times when plasma concentrations are rising or falling. Careful clinical observations accompanied by concomitant evaluation of plasma dopa levels have shown that the clinical assumptions regarding the nature of dyskinesias (e.g. whether they are peak dose, diphasic, or other) may, at times, be incorrect.

Support for continuous dopaminergic stimulation in PD comes from the work discussed by Dr. Mouraidian above, as well as from clinical studies. After two or more years on sustained dopaminergic drugs such as bromocriptine or lisuride (alone or in combination with levodopa) or on controlled release levodopa in advanced PD, there are fewer dyskinesias than on standard levodopa. It remains to be established whether the response fluctuations on standard levodopa therapy may also benefit from DA agonists and long acting levodopa compounds.

The various methods of continuous dopaminergic stimulation were reviewed by Dr. Sage. Continuous duodenal or jejunal levodopa delivery produces improvement in parkinsonian fluctuations and in the involuntary movements such as chorea and dystonia. This benefit has been sustained for more than four years in some patients. Around the clock infusion may shift the levodopa response curve to the right resulting in an increased requirement for levodopa to sustain the same antiparkinsonian 
benefit. Continuous daytime-only infusions (17-18 hours daily) has resulted in a gradual decline in levodopa requirements to maintain the same antiparkinsonian benefit. The plasticity emphasized by the work of Mouraidian and colleagues has also been seen here with an increase in dyskinesia threshold and an increase in the window of benefit between achieving the "on" state and the onset of chorea. The drawbacks of using levodopa for continuous infusion therapy, such as the large volumes of water required for solubility and competition with large neutral amino acids for absorption from the gut and delivery across the bloodbrain barrier may be overcome with alternative treatments.

Dr. Anthony Lang from the University of Toronto discussed experimental symptomatic therapy and protective therapy in $\mathrm{PD}$. Lisuride, a water soluble ergot dopamine agonist has been used extensively for subcutaneous infusion studies. Impressive results have been reported by some groups using the dopamine agonist apomorphine, both by continuous subcutaneous infusion and intermittent subcutaneous injection. The dopamine agonist PHNO is absorbed transdermally and antiparkinsonian effects in animals are seen when the drug is applied as a skin patch. Although this drug has been withdrawn, there is considerable hope for this approach with future agents. "Pro drugs" such as levodopa methyl ester and the catecholmonoester NB355 are other promising alternatives. A single patient in Australia has been treated with an intraventricular infusion of dopamine. This treatment was effective; however a significant observation was that symptoms poorly responsive to oral levodopa responded no better to this approach. Finally, striatal polymeric implants have been shown to have the potential to release dopamine over very prolonged periods of time. This experimental approach may eventually be applicable to human patients.

"Atypical" neuroleptics such as clozapine and remoxipride may have the potential for controlling psychiatric complications in levodopa-treated parkinsonian patients with less potential for aggravating the underlying parkinsonism than standard neuroleptic drugs. A small number of catechol-O-methyl transferase inhibitors are currently being studied. Peripherally-acting agents may allow more levodopa to access the brain while those with central effects may additionally prolong the duration of action of dopamine formed from levodopa. Promising potentiating effects on levodopa come from animal studies using CCK-B antagonist, L 365-260.

Transplantation therapy for Parkinson's disease remains an exciting field. The initial enthusiasm for adrenal medullary transplantation have been tempered by more recent studies. Only mild beneficial effects are usually obtained and these seem to wane with time. As currently performed, this approach, should not be adopted into the therapeutic armamentarium for PD. Recent results of fetal nigral cell transplantation, especially those from Sweden, are promising. Not only has clinical improvement been demonstrated, but there has been evidence of viability and production of dopamine by the graft, seen on fluorodopa PET scanning.

Dr. Lang also presented brief review of protective drug therapy for PD. Evidence for both exogenous and endogenous oxidatively-mediated mechanisms contributing to and possibly causing nigral dopaminergic cell loss in PD were discussed. These findings led to a multi-institutional trial designed to assess the ability of the selective MAO-B inhibitor, Deprenyl and the non-selective free radical scavenger, alpha tocopherol, to slow the progression of $\mathrm{PD}$, as evidenced by a delay in the time until levodopa is required for the symptomatic treatment. The results of two such trials, one by Tetrud and Langston, and the other by the Parkinson Study Group (DATATOP) were published late in 1989. Both revealed that patients treated with Deprenyl had a significant prolongation (approximately one year) to the time that their disability required additional levodopa therapy. The smaller study failed to show any symptomatic effects of Deprenyl therapy while the DATATOP Study showed a small improvement in symptoms which was insufficient to explain the major differences in the rate of disability between the Deprenyl treated and non treated groups. Additional analysis of the data supports the conclusion that Deprenyl affects the process of PD rather than simply improving symptoms. Comparing those Deprenyl-treated patients whose signs of parkinsonism remained unchanged or worsened after one to three months of therapy (i.e., no positive wash-in effect) to the non-Deprenyl-treated subjects who improved during same time (i.e., a positive symptomatic "wash-in" effect) the difference between the proportion of subjects reaching end-point for the two groups remained significantly in favour or Deprenyl therapy. Several persisting unanswered or lingering questions regarding the DATATOP trial were reviewed. Additional analysis of existing data and the results of alterations in the protocol carried out subsequent to the discovery of the marked effect of Deprenyl will hopefully resolve many of these issues. Further studies utilizing similar MAO-B inhibitors which lack certain pharmacological properties of Deprenyl (particularly the metabolism to amphetamine derivatives) also may assist in resolving some of these issues.

Dr. C. Tanner from Chicago and from the Clinical Center for Movement Disorders, San Jose, California discussed some new frontiers in the management of mild to moderate PD. Mildly affected patients are those who are able to maintain independent function at work and in daily activities without the need for antiparkinson therapy. In these individuals, the major goal of treatment is to delay the progression of the disease with drugs such as Deprenyl. The role of other antioxidant drugs such as alpha tocopherhol remains to be established. Moderately affected patients have more severe functional impairment which is sufficient to interfere with employment or independence in activities of daily living and mild alteration in postural reflexes. In addition delaying disease progression, additional goals of treatment in this group include enhancing daily function and avoiding the premature development of levodopa-related adverse effects. Initially, anticholinergics and/or amantadine may improve function sufficiently to delay the need for more potent therapy. Once the disability is sufficient to require the more potent agents, treatment strategies should include a consideration of how to delay adverse effects. Monotherapy with dopamine receptor agonists typically results in fewer dykinesias and fluctuations; however initial efficacy is often not sustained. When low doses of levodopa are combined with an agonist such as bromocriptine or lisuride, the development of fluctuations and dyskinesias are delayed compared to treatment with higher doses of levodopa alone. The occurrence of psychiatric side effects is similar in the two groups. Preliminary studies suggest that compared with 
standard levodopa, controlled release levodopa used in early stages of PD results in few fluctuations and dyskinesias after two years. These findings support the hypothesis that pulsatile oral levodopa therapy causes pharmacodynamic changes which result in some of these complications. Several large scale studies designed to study this question are currently in progress.

Dr. O. Kofman of University of Toronto, reviewed the current controversy over whether the long term affects of Deprenyl were achieved through a neuroprotective action or simply alleviation of the dopaminergic deficit with a consequent improvement in symptoms. (See editorial comment elsewhere in this issue of the Journal.) These presentations were followed by a lively debate on the protective versus symptomatic effects of Deprenyl. 